\title{
Induction of cell death after localization to the host cell mitochondria by the Mycobacterium tuberculosis PE_PGRS33 protein
}

\begin{abstract}
Correspondence
Nathalie Cadieux
\end{abstract}

ncadieux@aeras.org

Received 22 June 2010

Revised 18 October 2010

Accepted 12 November 2010

\author{
Nathalie Cadieux, ${ }^{1} \dagger$ Marcela Parra, ${ }^{1}$ Hannah Cohen, ${ }^{1} \ddagger$ Dragan Maric, ${ }^{2}$ \\ Sheldon L. Morris ${ }^{1}$ and Michael J. Brennan ${ }^{1} \dagger$
${ }^{1}$ Center for Biologics Evaluation and Research, Food and Drug Administration, Bethesda, MD, USA
${ }^{2}$ National Institute of Neurological Disorders and Stroke, Flow Cytometry Core Facility, NIH, Bethesda, MD, USA

\begin{abstract}
PE_PGRS33 is the most studied member of the unique PE family of mycobacterial proteins. These proteins are composed of a PE domain (Pro-Glu motif), a linker region and a PGRS domain (polymorphic GC-rich-repetitive sequence). Previous studies have shown that PE_PGRS33 is surface-exposed, constitutively expressed during growth and infection, involved in creating antigenic diversity, and able to induce death in transfected or infected eukaryotic cells. In this study, we showed that PE_PGRS33 co-localizes to the mitochondria of transfected cells, a phenomenon dependent on the linker region and the PGRS domain, but not the PE domain. Using different genetic fusions and chimeras, we also demonstrated a direct correlation between localization to the host mitochondria and the induction of cell death. Finally, although all constructs localizing to the mitochondria did induce apoptosis, only the wild-type PE_PGRS33 with its own $\mathrm{PE}$ domain also induced primary necrosis, indicating a potentially important role for the PE domain. Considering the importance of primary necrosis in Mycobacterium tuberculosis dissemination during natural infection, the PE_PGRS33 protein may play a crucial role in the pathogenesis of tuberculosis.
\end{abstract}

\section{INTRODUCTION}

Approximately one third of the world's population is infected with Mycobacterium tuberculosis, the causative agent of tuberculosis. With nearly 9 million new cases and almost 2 million deaths reported annually, this pathogen remains a major cause of morbidity and mortality worldwide (WHO, 2008). However, despite its global public health significance, the molecular mechanisms of pathogenesis for M. tuberculosis have not been well defined. Interestingly, nucleotide sequence analysis of the $M$. tuberculosis genome revealed the existence of unique multigene families (the PE and PPE genes) which account for approximately $10 \%$ of its coding capacity and are characterized by a high GC content and extensively

tPresent address: AERAS Global TB Vaccine Foundation, Rockville, MD, USA.

$\ddagger$ Present address: Division of Pharmaceutical Sciences, University of Wisconsin-Madison, Madison, WI, USA.

Abbreviations: BMMO, bone marrow-derived macrophages; IFA, immunofluorescence assay; $\mathrm{mAb}$, monoclonal antibody; PI, propidium iodide; RD, human rhabdomyosarcoma.

Three supplementary figures are available with the online version of this paper. repetitive homologous sequences (Camus et al., 2002; Cole et al., 1998). Members of the PE family have a characteristic Pro-Glu (PE) motif near the beginning of their conserved $\mathrm{N}$-terminal domain, and can be further subdivided into the PE (34 members) and PE_PGRS (65 members) subfamilies on the basis of their C-terminal domain (Brennan et al., 2005; Gey van Pittius et al., 2006; Gordon et al., 1999). The PE_PGRS proteins have three distinct regions: an $\mathrm{N}$-terminal $\mathrm{PE}$ domain, a linker region and a highly hydrophobic PGRS (polymorphic GC-richrepetitive sequence) domain containing a variable number of GGA-GGX repeats (Brennan et al., 2001; Poulet \& Cole, 1995). Although the exact roles of the PE_PGRS proteins in M. tuberculosis biology and pathogenesis have not been clearly elucidated, previous studies have shown that they are surface-exposed and available for interactions with the host (Delogu et al., 2004), differentially expressed during growth and infection (Delogu et al., 2006; Dheenadhayalan et al., 2006b; Espitia et al., 1999; Flores \& Espitia, 2003; Ramakrishnan et al., 2000; Talaat et al., 2004) and involved in creating antigenic variability (Karboul et al., 2008; Talarico et al., 2005, 2007, 2008).

A widely studied member of this family of $M$. tuberculosis proteins is PE_PGRS33, which is encoded by the $R v 1818 \mathrm{c}$ 
gene. A potential role for PE_PGRS33 in pathogenesis was initially shown when a BCG transposon mutant displaying less infectivity in J774 cells and bone marrow-derived macrophages (BMMO) was found to have an insertion in the homologue of $R v 1818 c$ (Brennan et al., 2001). Further studies have revealed that PE_PGRS33 is constitutively expressed under different growth conditions as well as during infection of BMMO and mice (Dheenadhayalan et al., 2006b). In addition, BMMO infected with a recombinant Mycobacterium smegmatis strain expressing the full-length PE_PGRS33 protein underwent necrosis, which resulted in dissemination and enhanced survival of the bacteria (Dheenadhayalan et al., 2006a). More recent studies have demonstrated that recombinant PE_PGRS33 protein, as well as an M. smegmatis strain overexpressing PE_PGRS33, induced apoptosis of RAW264.7 cells and that Jurkat $\mathrm{T}$ cells transfected with a PE_PGRS33-EGFP fusion also became apoptotic (Balaji et al., 2007; Basu et al., 2007). In both studies, mitochondrial intermediates were implicated and in the latter, Balaji and collaborators also observed partial co-localization of the transfected $\mathrm{PE}_{-}$ PGRS33-EGFP with the host mitochondria. Interestingly, mitochondrial damage is central to both apoptosis and necrosis and has been shown to be extremely important in determining the outcome of macrophage infection with $M$. tuberculosis (Abarca-Rojano et al., 2003; Duan et al., 2002; Gan et al., 2005). Furthermore, the type of cell death induced determines the fate of the bacteria within the host (Porcelli \& Jacobs, 2008). Indeed, avirulent M. tuberculosis strains such as H37Ra can induce apoptosis of macrophages which leads to containment and killing of the bacteria, as well as priming of $\mathrm{T}$ cell responses (Chen et al., 2006; Fratazzi et al., 1997; Schaible et al., 2003). In contrast, virulent strains such as $\mathrm{H} 37 \mathrm{Rv}$ often induce macrophage necrosis leading to escape and dissemination of the bacteria, which is clearly advantageous to the pathogen (Chen et al., 2006; Fratazzi et al., 1997; Park et al., 2006). For these reasons, defining the parameters which allow PE_PGRS33 to co-localize to the mitochondria of host cells and identifying the type of cell death induced should enhance our understanding of the pathogenic interactions between mycobacteria and mitochondria.

\section{METHODS}

Bacteria, cell lines and culture conditions. Escherichia coli DH5 $\alpha$ was used as the bacterial host for cloning purposes and was grown in Luria-Bertani medium supplemented with $100 \mu \mathrm{g}$ ampicillin $\mathrm{ml}^{-1}$ when harbouring a plasmid. Human rhabdomyosarcoma (RD) cells were obtained from the American type culture collection (ATCC) and were grown in RPMI medium containing $10 \%$ fetal calf serum, $2 \mathrm{mM}$ L-glutamine, $10 \mathrm{mM}$ HEPES, $1 \mathrm{mM}$ sodium pyruvate and nonessential amino acids. The T-REx (Invitrogen) RD cell lines created in this study were grown in RPMI medium containing tetracyclinescreened fetal calf serum (HyClone) and supplemented with $10 \mu \mathrm{g}$ blasticidin $\mathrm{ml}^{-1}$ and $200 \mu \mathrm{g}$ zeocin $\mathrm{ml}^{-1}$ (Invitrogen). Protein expression by the stable cell lines was induced by the addition of $5 \mu \mathrm{g}$ tetracycline $\mathrm{ml}^{-1}$.
DNA constructs. The DNA constructs used for transient transfections were prepared in the mammalian expression vector pJW4303. The constructs used to create the stable T-REx RD cell lines were prepared in the mammalian inducible expression vector pcDNA 4/TO (Invitrogen). In all cases, the inserts (full-length genes or individual domains) were prepared by adding a HindIII site at the $5^{\prime}$ end and a BamHI site at the $3^{\prime}$ end, using PCR as described previously, except the PE_PGRS1 constructs for which an AvrII site was used at the 3' end because of a pre-existing BamHI site within the coding sequence of that gene (Cadieux \& Kadner, 1999). Additional restriction sites (NruI, MluI or EcoRV, depending on the constructs) were also engineered internally from the HindIII and BamHI/AvrII sites (Fig. 1a), as well as a removable stop codon at the $3^{\prime}$ end. All inserts or domains were first cloned into pJW4303 using HindIII and BamHI or AvrII, before being combined using the internal restriction sites, which allowed the removal of the stop codon from the first insert and the fusion of the second insert, in-frame. In the case of the PE_PGRS33/PE_PGRS1 chimeras which resulted from the sequential fusion of three inserts, the restriction site between the linker regions and the PGRS domains had to be removed by site-directed mutagenesis using a two-step PCR protocol because its presence disrupted proper localization of the reconstituted control PE_PGRS33 protein. DNA manipulations followed standard protocols and all inserts were verified by complete DNA sequencing at the CBER sequencing core facility. See Table 1 for a complete list and description of the constructs prepared in this study.

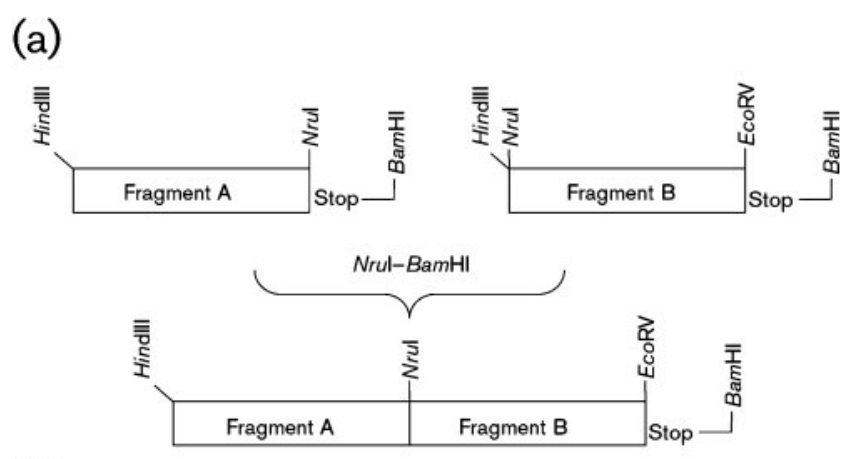

(b)

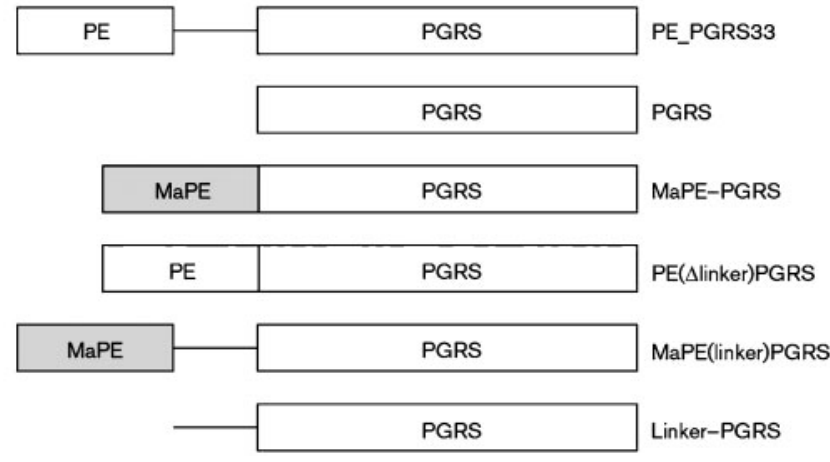

Fig. 1. Protein fusions constructed in this study. (a) Schematic of the method used to construct the fusions. Inserts were engineered to have a HindIII at their $5^{\prime}$ end and a BamHI site at their $3^{\prime}$ end, for cloning into pJW4303. Additional sites such as Nrul or EcoRV were included internally, as well as a removable stop codon to allow inserts to be fused together, in-frame. (b) Schematic of the fusions constructed using this method. 
Table 1. Constructs prepared and used in this study

\begin{tabular}{|c|c|}
\hline Construct & Description \\
\hline PE_PGRS33 & Full-length PE_PGRS33 \\
\hline PGRS & PGRS domain of PE_PGRS33 (nt 424-1597) \\
\hline linker-PGRS & Linker and PGRS domain of PE_PGRS33 (nt 301-1497) \\
\hline MaPE(linker)PGRS & Linker and PGRS domain of PE_PGRS33 fused to MaPE \\
\hline $\operatorname{PE}(\Delta$ linker $)$ PGRS & PGRS domain of PE_PGRS33 fused directly to its PE domain (removing the linker region) \\
\hline PE_PGRS18 & Full-length PE_PGRS18 \\
\hline PE_PGRS24 & Full-length PE_PGRS24 \\
\hline PE1-linker1 $\Delta-$ PGRS1 & PE_PGRS1 without the first 7 aa of its linker \\
\hline PE33-linker1-PGRS33 & PE of PE_PGRS33, linker of PE_PGRS1, PGRS of PE_PGRS33 \\
\hline PE1-linker33-PGRS1 & PE of PE_PGRS1, linker of PE_PGRS33, PGRS of PE_PGRS1 \\
\hline PE33-linker33-PGRS1 & PE of PE_PGRS33, linker of PE_PGRS33, PGRS of PE_PGRS1 \\
\hline
\end{tabular}

Transient transfections and creation of stable T-REx RD cell lines. For the transient transfections, RD cells were transfected with $1 \mu \mathrm{g}$ of the different DNA constructs using Lipofectamine 2000 (Invitrogen) following the manufacturer's instructions. Protein expression was evaluated $24 \mathrm{~h}$ after transfection by direct fluorescence microscopy (constructs containing EGFP) or after immunofluorescence assay (IFA) or Western blot. For the creation of stable RD cell lines expressing the different constructs used in this study, the T-REx inducible mammalian expression system was used (Invitrogen). A suppressor cell line was first created by transfecting the pcDNA6/TR plasmid, carrying the tetracycline repressor, into the original RD cell line using Lipofectamine 2000. The resulting transfectants were then cultured in the presence of $10 \mu \mathrm{g}$ blasticidin $\mathrm{ml}^{-1}$ in order to select for the repressor. Potential clones were picked, expanded and tested for their ability to repress the expression of a test protein cloned into the pcDNA 4/TO plasmid, carrying the tetracycline operator. The cell line giving the lowest expression background and the best increase in expression when induced with $5 \mu \mathrm{g}$ tetracycline $\mathrm{ml}^{-1}$ was chosen as the T-REx RD repressor cell line. For the stable T-REx RD inducible cell lines, the T-REx RD repressor was transfected with the different constructs cloned in the pcDNA 4/TO plasmid. The resulting transfectants were grown in the presence of $10 \mu \mathrm{g}$ blasticidin $\mathrm{ml}^{-1}$ (repressor) and $200 \mu \mathrm{g}$ zeocin $\mathrm{ml}^{-1}$ (operator). Potential clones were picked, expanded and tested for protein expression by IFA, $24 \mathrm{~h}$ after induction with $5 \mu \mathrm{g}$ tetracycline $\mathrm{ml}^{-1}$. The best expressors were selected and subjected to additional rounds of selection in order to obtain cell lines with as many expressing cells as possible.

\section{Immunoassays}

Immunofluorescence assay. At different times following induction, cells grown on glass coverslips were stained for $30 \mathrm{~min}$ at $37^{\circ} \mathrm{C}$ with $50 \mathrm{nM}$ MitoTracker Red CMXRos (Invitrogen) before being fixed with $4 \%$ paraformaldehyde in PBS for $20 \mathrm{~min}$ at room temperature. The cells were then permeabilized in PBS/ $0.2 \%$ Triton X-100 for $5 \mathrm{~min}$ and blocked for $1 \mathrm{~h}$ at room temperature in PBS/3\% BSA containing $10 \%$ normal goat serum. Subsequently, the samples were incubated with ascites from the PE_PGRS33-specific monoclonal antibody 7C4.1F7 diluted $1: 1000$ in $\mathrm{PBS} / 3 \% \mathrm{BSA}$ for $1 \mathrm{~h}$ before being washed three times in PBS and incubated in goat anti-mouse AlexaFluor 488-conjugated secondary antibody (Invitrogen) diluted $1: 1000$ in $\mathrm{PBS} / 3 \%$ BSA for an additional hour. After three more washes in PBS, the coverslips were mounted on slides using
Fluoromount-G (SouthernBiotech) and observed on a Nikon OptiPhot fluorescent microscope using a B-1A filter cube for the green fluorescence and a G-1B filter cube for the red fluorescence. Images were captured with a SPOT RT colour camera (Diagnostic Instruments).

Western blots. The T-REx RD cells were induced for $24 \mathrm{~h}$ and collected; their proteins were separated onto NuPAGE 10\% Bistris gels (Invitrogen) before being transferred onto nitrocellulose membranes using the buffer system described initially by Towbin et al. (1979). The membranes were blocked in PBS/5\% non-fat dried milk for $1 \mathrm{~h}$ at room temperature before being incubated in the PE_PGRS33-specific 7C4.1F7 monoclonal antibody diluted 1:5000 in the same buffer for $1 \mathrm{~h}$. After three washes in PBS/0.02\% Tween 20, the membranes were incubated in goat anti-mouse alkaline phosphataseconjugated secondary antibody (Jackson ImmunoResearch Laboratories), diluted $1: 3000$ in PBS $/ 5 \%$ milk for $1 \mathrm{~h}$. After three more washes in PBS/0.02\% Tween 20, the blots were developed using the colorimetric substrate NBT/BCIP (KPL).

Internal staining by flow cytometry. Cells were grown in 6-well plates, induced for different times, collected by trypsinization and fixed in $2 \%$ paraformaldehyde in PBS for $20 \mathrm{~min}$ at room temperature. After being washed in FACS buffer (PBS/2\% FBS), $5 \times 10^{5}$ cells were resuspended in $100 \mu \mathrm{l}$ permeabilization buffer (DPBS with $1 \mathrm{mM} \mathrm{Ca}^{2+}, 0.5 \mathrm{mM} \mathrm{Mg}^{2+}, 10 \mathrm{mM}$ HEPES, $1 \%$ FCS, $0.1 \%$ saponin), containing $10 \%$ normal goat serum and $10 \mu \mathrm{g} \mathrm{Fc}$ block $\mathrm{ml}^{-1}$ (BD Biosciences). Following a $30 \mathrm{~min}$ incubation on ice, the PE_PGRS33-specific 7C4.1F7 monoclonal antibody (mAb) was added at a final concentration of $1 \mu \mathrm{g} \mathrm{ml}^{-1}$ for $30 \mathrm{~min}$ on ice. The samples were then washed twice with permeabilization buffer, incubated in $100 \mu \mathrm{l}$ permeabilization buffer containing $2 \mu \mathrm{g} \mathrm{ml} \mathrm{m}^{-1}$ goat anti-mouse AlexaFluor 488-conjugated secondary antibody for $30 \mathrm{~min}$ on ice, and washed twice with permeabilization buffer before being fixed in $0.5 \%$ paraformaldehyde in PBS. The samples were later analysed on an LSRII flow cytometer (BD Biosciences). The data were collected by using the FACS DIVA software and analysed by using the FlowJo software.

\section{Cell death analyses}

Flow cytometric cell death analysis (Hoechst 33342/YO-PRO-1/PI staining). At different times following induction, the T-REx RD cell 
lines were collected and pooled with the culture supernatants containing dead or dying cells. The T-REx RD cells were then adjusted to $7.5 \times 10^{5}$ cells in $500 \mu \mathrm{PBS} / 1 \mathrm{mg} \mathrm{BSA} \mathrm{ml} \mathrm{m}^{-1}$ and were stained for $20 \mathrm{~min}$ at room temperature with $1 \mu \mathrm{l}$ Hoechst 33342, $1 \mu \mathrm{l}$ YO-PRO- 1 and $0.5 \mu \mathrm{l}$ propidium iodide (all provided in the Vybrant Apoptosis Assay kit \#7; Invitrogen). The samples were run on a FACVantage SEM flow cytometer (BD Biosciences) and the data were acquired and analysed by using the CellQuest Acquisition and Analysis Software (BD Biosciences).

Mitochondrial membrane potential measurements (JC-1 staining). The T-REx RD cell lines were grown, induced and collected as described above. The cells were adjusted to $7.5 \times 10^{5} \mathrm{ml}^{-1}$ and stained with $1 \mu \mathrm{g} \mathrm{JC}-1$ reagent $\mathrm{ml}^{-1}$ (Invitrogen) for $15 \mathrm{~min}$ at $37^{\circ} \mathrm{C}$ before being washed and resuspended in PBS/1 mg BSA ml ${ }^{-1}$. The samples were run on a FACVantage SEM flow cytometer (BD Biosciences) and the data were acquired and analysed by using the CellQuest Acquisition and Analysis Software (BD Biosciences).

\section{RESULTS}

\section{Localization of the PE_PGRS33 protein in transfected eukaryotic cells}

In a previous study, our group used EGFP fusions to examine the localization of PE_PGRS33 in transfected eukaryotic cells (Brennan \& Delogu, 2002). We found that the PE domain of PE_PGRS33 fused to EGFP showed a diffuse pattern of fluorescence throughout the cell, whereas the full-length PE_PGRS33 showed foci of dense protein accumulation, generally in the perinuclear area of the cells. In the present study, we used a PE_PGRS33-specific mAb (7C4.1F7) prepared in our laboratory to localize PE_PGRS proteins directly without having to fuse them to EGFP. This $\mathrm{mAb}$ recognizes the PGRS domain of PE_PGRS antigens (Abdallah et al., 2009; Singh et al., 2008). Using this $\mathrm{mAb}$, the patterns of fluorescence seen when a plasmid expressing the PE_PGRS33 protein was transfected into RD cells were different from those previously observed with the EGFP fusion, and they were found to correspond to the mitochondria of host cells. Cells shown in Fig. 2, were transfected with either the vector alone (Fig. 2a) or PE_PGRS33 (Fig. 2b and c) and stained with the mitochondria-specific dye MitoTracker Red before being treated with the PE_PGRS33-specific mAb 7C4.1F7. Cells transfected with the vector alone did not bind to the $\mathrm{mAb}$, whereas cells expressing PE_PGRS33 did, showing the specificity of this mAb. Merging of the mAb- and MitoTracker-stained images clearly showed co-localization between PE_PGRS33 and the mitochondria of transfected host cells (Fig. 2b and c, right panels). Interestingly, in many cases, the mitochondria of cells expressing PE_PGRS33 appeared swollen compared with the mitochondria of untransfected cells, presumably the result of mitochondria-PE_PGRS33 interactions (Fig. 2c). Similar results were obtained with macrophage cell lines such as J774 and RAW264.7 cells (data not shown), but very low transfection efficiencies confounded experimentation with these cell lines.

\section{Regions of PE_PGRS33 required for co-localization to the mitochondria}

To determine which regions of PE_PGRS33 are necessary for its proper localization to the mitochondria of transfected host cells, different constructs were generated by using a method that allows the fusion of multiple inserts in-frame, as described in Methods and in Fig. 1(a). Since the PE_PGRS33-specific mAb 7C4.1F7 recognizes the PGRS domain of PE_PGRS33 and not the PE region (data
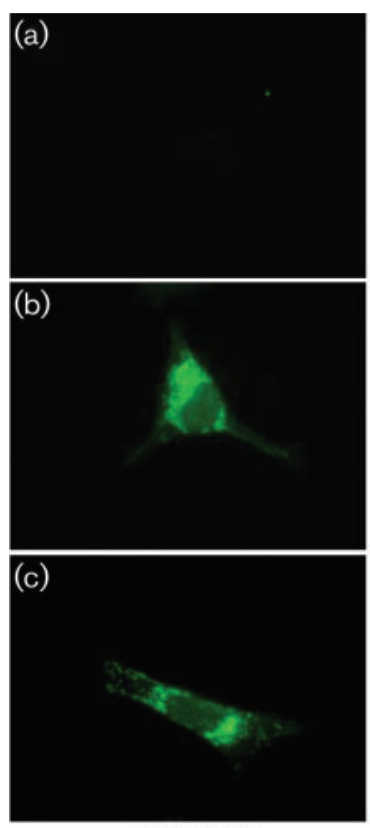

$7 \mathrm{C} 4 \mathrm{mAb}$
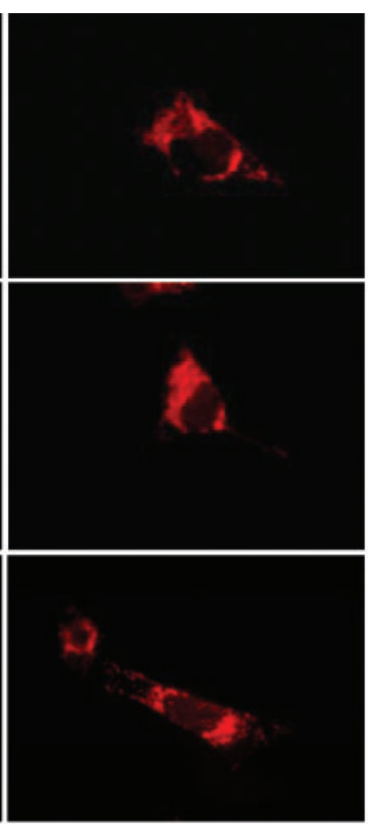

MitoTracker Red

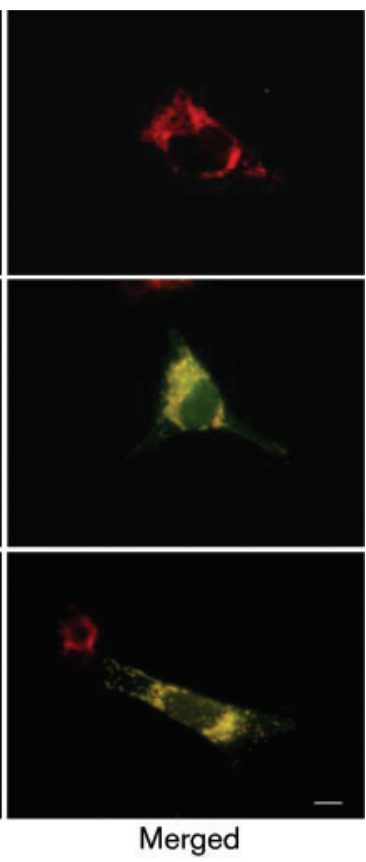

Fig. 2. PE_PGRS33 co-localizes to the mitochondria of transfected host cells. RD cells were transfected with the pJW4303 vector (a) or PE_PGRS33 (b and c). Cells stained with the PE_PGRS33-specific mAb 7C4.1F7 (left) and the mitochondria-specific dye MitoTracker Red (centre) and the merged images (right) are shown. Magnification, $\times 1000$; bar, $10 \mu \mathrm{m}$. 
not shown), that domain was present in all the constructs included in this study (Fig. 1b).

The role of the PGRS domain in the localization of the PE_PGRS33 protein was evaluated first. Unlike the full-length PE_PGRS33 which localizes to the mitochondria (Fig. 3a) the PGRS domain by itself showed a diffuse pattern of fluorescence throughout the cell (Fig. 3b). The PGRS domain was then fused directly to MaPE, a PE protein from Mycobacterium avium, which was recently shown to be a potential new vaccine candidate (Parra et al., 2006). Interestingly, the MaPE-PGRS fusion also did not localize to the mitochondria of the transfected host cells (Fig. 3c), despite the fact that MaPE is $63 \%$ identical to the PE domain of PE_PGRS33. Since the MaPE-PGRS fusion did not contain the 41 amino acid linker region normally found between the PE and PGRS domains of PE_PGRS33, two more fusions were prepared to try to determine if that region had any importance in the proper localization of PE_PGRS33. In the first construct, the PE domain of PE_PGRS33 was fused directly to its PGRS domain, thereby removing this 41 amino acid region, resulting in a $\mathrm{PE}(\Delta$ linker $)$ PGRS protein. In the second construct, the linker region was added between MaPE and PGRS, essentially creating an artificial PE_PGRS protein, namely MaPE(linker)PGRS. As seen in Fig. 3(d), the PE( $\Delta$ linker)PGRS protein did not localize to the mitochondria of transfected host cells but the MaPE(linker)PGRS protein did (Fig. 3e), indicating that the linker region is essential for this localization phenomenon. Finally, in order to determine if the PE domain played any role in the localization to the mitochondria, a construct was generated that consisted exclusively of the linker region and the PGRS domain (linkerPGRS). Surprisingly, this construct showed a mixed phenotype, with diffuse fluorescence throughout the cells, but also co-localization with the mitochondria (Fig. 3f), despite the absence of the PE domain.

\section{Creation and characterization of tetracycline- inducible stable cell lines}

To improve the consistency of the transfection experiments, stable transfectants were created using the tetracyclineinducible T-REx system from Invitrogen. After multiple rounds of selection to increase the percentage of expressing cells, the cell lines were induced for $24-48 \mathrm{~h}$ with tetracycline and tested by IFA with the PE_PGRS33-specific $\mathrm{mAb} 7 \mathrm{C} 4.1 \mathrm{~F} 7$ to confirm that they all demonstrated the expected pattern of fluorescence (data not shown).

To study the kinetics of protein expression following induction with tetracycline, duplicate cell samples were collected, permeabilized and stained with the PE_PGRS33specific $\mathrm{mAb}$ 7C4.1F7 at different time points and analysed by flow cytometry. As seen in Supplementary Fig. S1(a) (available with the online version of this paper), all cell lines expressed fusion proteins at similar levels $(\sim 68-78 \%$ expressors) with the exception of the $\mathrm{PE}(\Delta$ linker)PGRS cell line ( $\sim 55 \%$ expressors). Additionally, in order to ensure that all the constructs expressed by the stable cell lines were of the correct size, cells were induced for $24 \mathrm{~h}$ and analysed by Western blot using the PE_PGRS33-specific mAb 7C4.1F7. The proteins expressed by the stable cell lines were all of the expected size, showed minimal degradation and the levels of expression at $24 \mathrm{~h}$ post-induction were consistent with the flow cytometry data (Supplementary Fig. S1b).

\section{Biological effects of the co-localization of PE_PGRS33 with the mitochondria of host cells}

The stable cell lines were used to investigate a possible correlation between the interaction of the PE_PGRS constructs with the mitochondria and the induction of cell death, and to determine which type of cell death was occurring. To get a precise assessment of cell death, the Vybrant Apoptosis Assay kit was used in conjunction with flow cytometry. This kit uses a combination of Hoechst 33342, YO-PRO-1 and propidium iodide (PI), three nuclear dyes with different permeability characteristics. Hoechst and PI can be used to determine the percentage of viable, apoptotic and necrotic cells based on total DNA content revealed by Hoechst staining and differential cell membrane permeability to PI, as described previously (Hillion et al., 2005). The YO-PRO-1/PI staining was used in this study mainly to identify and quantify the early apoptotic cells which have gained just enough membrane permeability to become YO-PRO-1-positive, but not enough to be PIpositive (data not shown). Dead cells become equally permeable to both, so it is not possible with this combination of dyes to determine if they underwent apoptosis followed by necrosis or just primary necrosis but it is possible to make that determination with the combined use of Hoechst and PI. Cells undergoing apoptosis and primary necrosis can be differentiated by how they label with those two dyes and many subpopulations along those pathways can be identified when gated appropriately. Viable cells stain brightly with Hoechst, which can enter intact cells, but they do not stain with PI, which can only enter cells that have a severely compromised plasma membrane. Apoptotic cells undergo DNA degradation and therefore show reduced Hoechst labelling because of their hypodiploid DNA content but increased PI labelling as they progress through the apoptotic pathway and their plasma membrane becomes more permeable. Conversely, cells that undergo primary necrosis do not show a loss of Hoechst labelling because their DNA does not become degraded, but they do show increased PI labelling as they become increasingly permeable. In this analysis, early necrotic cells were not taken into account because they are considered to be an intermediate state and can progress to either apoptosis or late necrosis. Examples of the results obtained with the TREx RD cell lines un-induced or at $72 \mathrm{~h}$ after induction are shown in Fig. 4(a). As expected, the vector control cell line and the PE( $\Delta$ linker)PGRS cell line, which does not colocalize to the mitochondria, showed mainly viable cells in un-induced samples and at $72 \mathrm{~h}$ post-induction. In contrast, the cell line expressing PE_PGRS33, which co-localizes to 


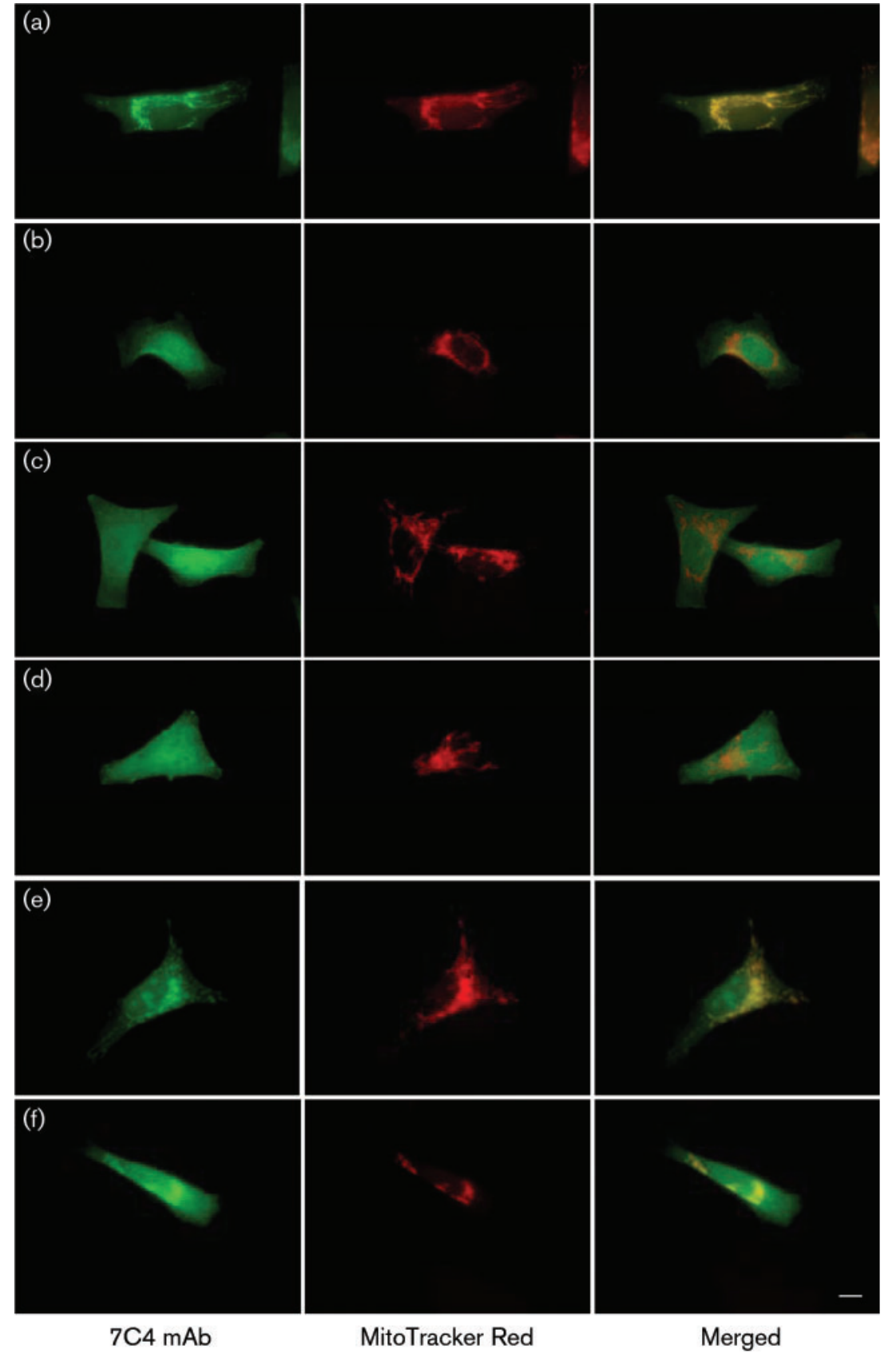

Fig. 3. Immunofluorescence showing the localization of the different protein fusions in transfected cells. RD cells were transfected with PE PGRS33 (a), PGRS (b), MaPE-PGRS (c), PE( $(\Delta$ linker)PGRS (d), MaPE(linker)PGRS (e) and linker-PGRS (f). Cells stained with the PE_PGRS33-specific mAb 7C4.1F7 (left) and the mitochondria-specific dye MitoTracker Red (centre) and the merged images (right) are shown. Magnification, $\times 1000$; bar, $10 \mu \mathrm{m}$. the mitochondria, showed a dramatic reduction in viable cells at $72 \mathrm{~h}$ post-induction and a corresponding increase in apoptotic and necrotic cells, especially the late stages. A complete time-course showing the number of viable, apoptotic and necrotic cells for all six T-REx RD cell lines is shown in Fig. 4(b). These results show that the vector control, as well as the two constructs, does not co-localize to the mitochondria and showed no significant loss of viability or increase in apoptosis and/or necrosis with time. In contrast, the three constructs that co-localize to the mitochondria of host cells did show a temporal reduction in viability and concurrent increase in cell death, although not to the same extent. Indeed, the cell lines expressing
MaPE(linker)PGRS and linker-PGRS showed a very similar and significant reduction in viability over time, with 51.3 and $54.5 \%$ viable cells remaining at $72 \mathrm{~h}$ post-induction $(P<0.001)$. Interestingly, most of the cell death was due to apoptosis (36.7 and $37.7 \%$, respectively) while the levels of primary necrosis observed with those cell lines were not significantly different (4.5 and $5.3 \%$ respectively). In sharp contrast, the cell line expressing the PE_PGRS33 protein showed an even more dramatic loss of viability with time, with viable cells as low as $4.5 \%$ at $72 \mathrm{~h}$ post-induction $(P<0.001)$. For this cell line, however, cell death was clearly due to both apoptosis ( $61.7 \%$ of cells) and primary necrosis (24.3\% of cells). The levels of apoptosis and necrosis 
(a)
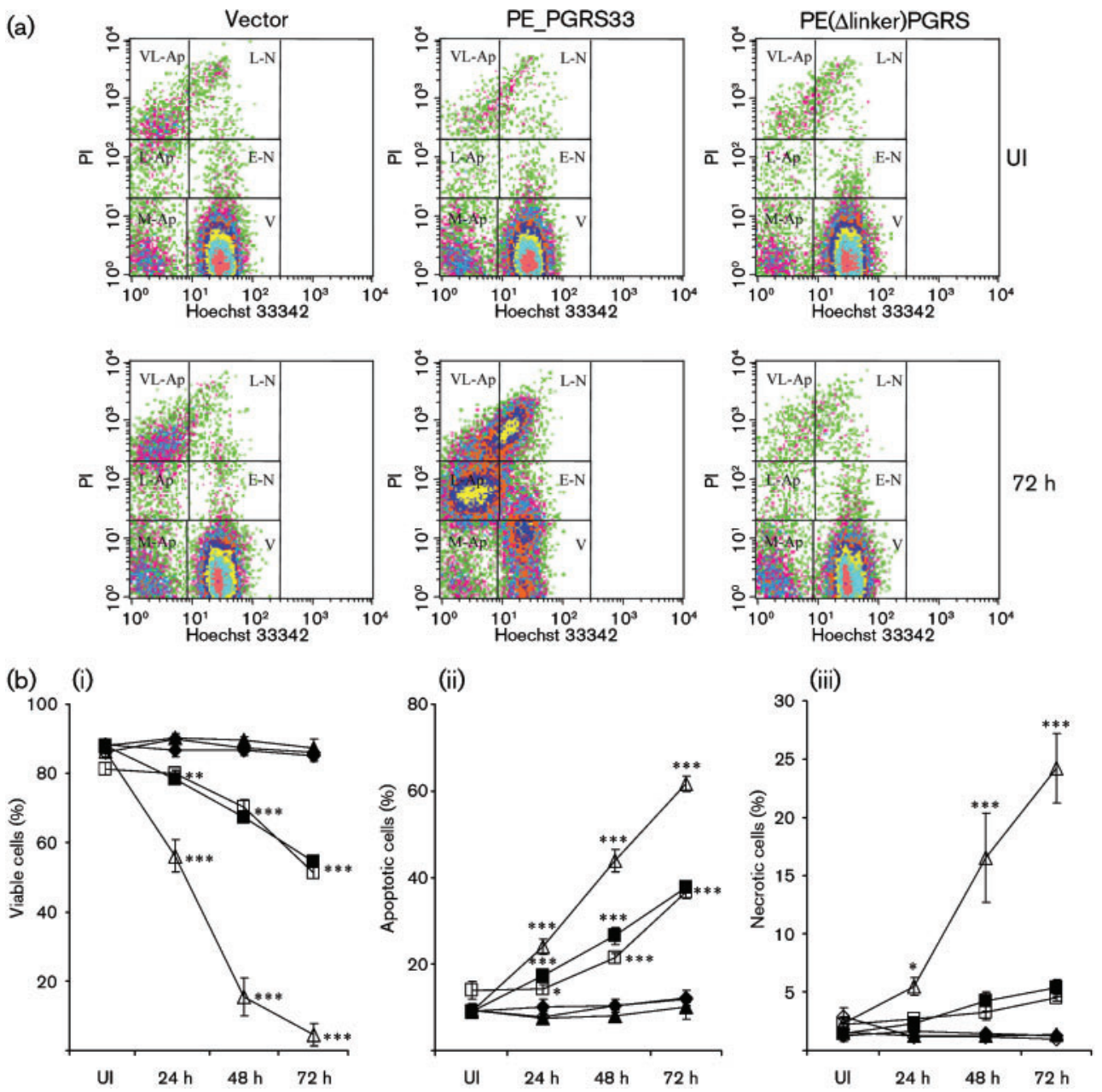

Fig. 4. Time-course showing the percentage of live, apoptotic and necrotic cells as determined by a combination of Hoechst 33342, YO-PRO-1 and PI staining, using the Vybrant Apoptosis Assay kit. T-REx RD cells were collected, stained and analysed by flow cytometry at different times following induction of protein expression. Based on differential staining with Hoechst and $\mathrm{PI}$, the cells were categorized into the following subpopulations: vital $(V)$ cells that stained brightly with Hoechst, but were negative for PI, indicating healthy plasma membranes; apoptotic (Ap) cells at mid (M-Ap), late (L-Ap) and very late (VL-Ap) stages of cell death that stained dimly with Hoechst because of their hypodiploid DNA content, but were progressively more PI-positive because of their increasingly compromised plasma membranes; and necrotic $(\mathrm{N})$ cells at early $(\mathrm{E}-\mathrm{N})$ and late $(\mathrm{L}-\mathrm{N})$ stages of cell death that exhibited Hoechst staining with an intensity comparable to that of viable cells but were increasingly PI-positive due to compromised plasma membranes. In some experiments, YO-PRO-1 was used as an additional DNA binding dye to reveal early apoptotic cells, which were otherwise indistinguishable from vital cells based on Hoechst and PI staining (data not shown). (a) Representative bivariate dot density plots of Hoechst and PI staining for the vector, PE_PGRS33 and PE( $\Delta$ linker)PGRS cell lines un-induced (UI) and at $72 \mathrm{~h}$ following induction. (b) The mean \pm SD of three independent experiments showing the percentage of viable (live cells minus early apoptotic cells; i), apoptotic (early, mid, late and very late; ii) and necrotic (late necrotic; iii) cells over time. Vector $(\boldsymbol{\Delta}), \operatorname{PE}$ P PGRS33 $(\triangle), \operatorname{PE}(\Delta$ linker)PGRS $(\diamond), \operatorname{MaPE}-\mathrm{PGRS}(\diamond), \mathrm{MaPE}($ linker $) \mathrm{PGRS}(\boldsymbol{\square})$ and linker-PGRS ( $\square$ ). Statistical analysis was performed using two-way ANOVA. ${ }^{*} P<0.05 ;{ }^{* \star} P<0.01 ;{ }^{* \star} P<0.001$.

detected for the PE_PGRS33-expressing cell line were significantly more than observed with the other two constructs $(P<0.001)$. These results confirm that there is a strong correlation between co-localization to the mitochondria and the induction of cell death, but they also suggest that the PE domain found in the wild-type PE_PGRS33 protein may mainly be responsible for the induction of primary necrosis.
To further correlate the induction of cell death to mitochondrial damage possibly caused by the different proteins binding to them, the very sensitive cationic dye JC-1 was used in conjunction with flow cytometry. The monomeric version of this dye fluoresces green, but its membrane-potential-dependent accumulation in intact mitochondria causes the formation of J-aggregates that fluoresce red. On examination by flow cytometry, cells with 
intact mitochondrial membrane potential will be red, cells in which the membrane potential has collapsed will be green and depolarizing cells with be both red and green. Examples of the results obtained with our cells lines are presented in Fig. 5. Once again, the vector control cell line and the cell line expressing the PE( $\Delta$ linker)PGRS construct (which does not bind to the mitochondria) showed mostly healthy, red cells both in un-induced samples and $48 \mathrm{~h}$ after the induction of protein expression. In contrast, cells expressing PE_PGRS33 showed a dramatic loss of viable (red) cells, a substantial increase in dead cells showing collapsed membrane potential (green) and a large number of cells with depolarizing mitochondria (red and green) at $48 \mathrm{~h}$ post-induction. In subsequent studies using the MaPE-PGRS-, MaPE(linker)PGRS- and linker-PGRSexpressing cell lines, a direct association was also shown between localization of mycobaterial protein to the mitochondria, mitochondrial damage and induction of cell death (data not shown).

\section{Localization of other PE_PGRS proteins in transfected host cells}

To determine if co-localization to the mitochondria is a characteristic shared by all members of the PE_PGRS family, other PGRS-containing proteins (PE_PGRS1, PE_PGRS18 and PE_PGRS24) were selected for further study based on a previous work where their expression pattern in culture and infected BMMO had been determined (Dheenadhayalan et al., 2006b). Unlike PE_PGRS33, none of the three additional PE_PGRS proteins tested co-localized fully with the mitochondria of host cells (Fig. 6). Each of them displayed a different pattern of fluorescence in transfected cells. PE_PGRS1 exhibited a diffuse pattern of fluorescence throughout the cells (Fig. 6a) while areas of protein accumulation, which were generally unrelated to the cellular mitochrondria, were observed with PE_PGRS18 expression (Fig. 6b). Finally, transfecting cells with PE_PGRS24 resulted in the mitochondria accumulating in the perinuclear area of the cells surrounding areas of protein accumulation, with partial co-localization between the two (Fig. 6c).

Since PE_PGRS1 showed a unique diffuse pattern of fluorescence that was not seen with any of the other PE_PGRS proteins, it was selected for further study. A sequence comparison between PE_PGRS1 and PE_PGRS33 revealed that they both have clearly delineated PE domains, linker regions and PGRS domains, with no significant area of sequence diversity that would explain such a difference in localization, except for a 7 amino acid stretch between the PE domain and linker region of PE_PGRS1 which is absent in PE_PGRS33 (Fig. 7a, underlined). To determine if this 7 amino acid segment prevents PE_PGRS1 from binding to the mitochondria of host cells, a construct was made lacking that region (Fig. 7b). The resulting construct, PE1linker1 $\Delta$-PGRS1 still did not localize to the mitochondria and showed the same diffuse pattern of fluorescence seen with the wild-type PE_PGRS1 (Supplementary Fig. S2a, available with the online version of this paper). Two more constructs were then prepared by switching the linker regions of PE_PGRS1 and PE_PGRS33 (Fig. 7b). The first one, PE1-linker33-PGRS1, also did not localize to the mitochondria, indicating that the linker33 alone is not responsible for mitochondrial localization (Supplementary Fig. S2b). Conversely, PE33-linker1-PGRS33 did localize to the mitochondria of transfected host cells, indicating that the 7 extra amino acids in the linker of PE_PGRS1 do not prevent it from binding to the mitochondria (Supplementary Fig. S2c). To further examine this localization process, additional chimeras were constructed by systematically swapping the PE domains, linker regions and PGRS domains of PE_PGRS1 and PE_PGRS33 (Fig. 7c). The results of these transfection experiments clearly indicate that
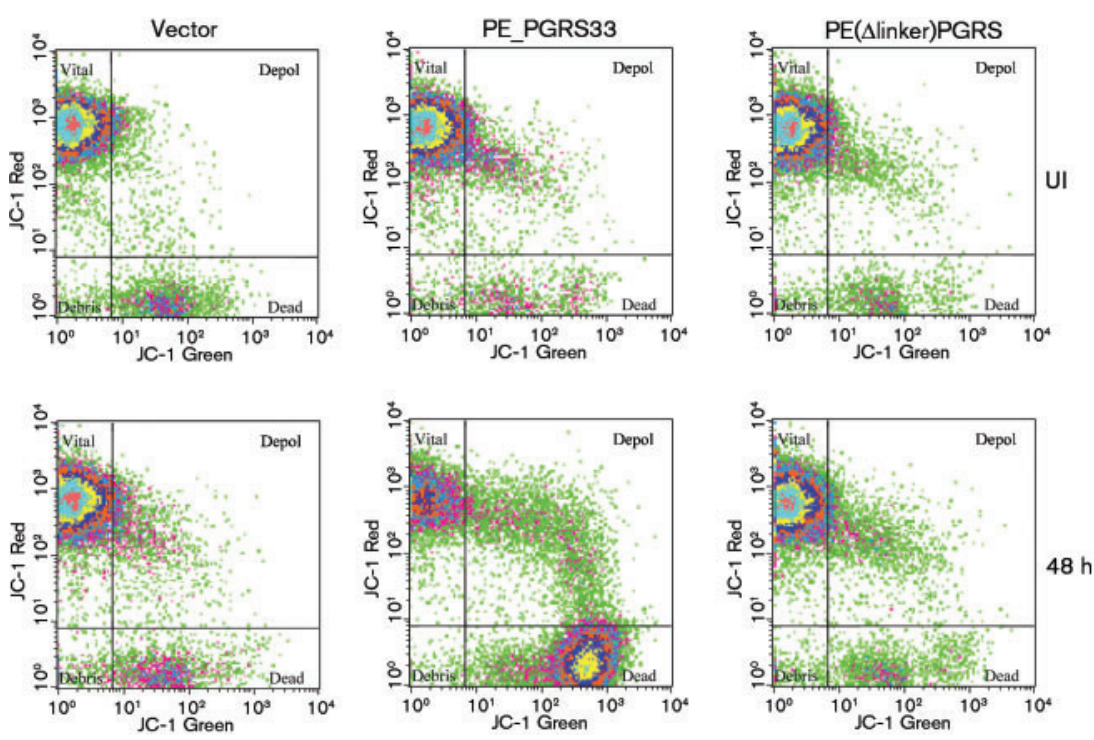

Fig. 5. Measurement of mitochondrial membrane potential using JC-1 staining. T-REx RD cells were collected, stained and analysed by flow cytometry at different times following induction of protein expression. Representative bivariate dot density plots for the vector, PE_PGRS33 and PE( $\Delta$ linker)PGRS cell lines un-induced $(\mathrm{UI})$ and $48 \mathrm{~h}$ after induction are shown. Viable cells with intact membrane potential are red, dead cells with collapsed membrane potential are green and depolarizing cells are both red and green. 


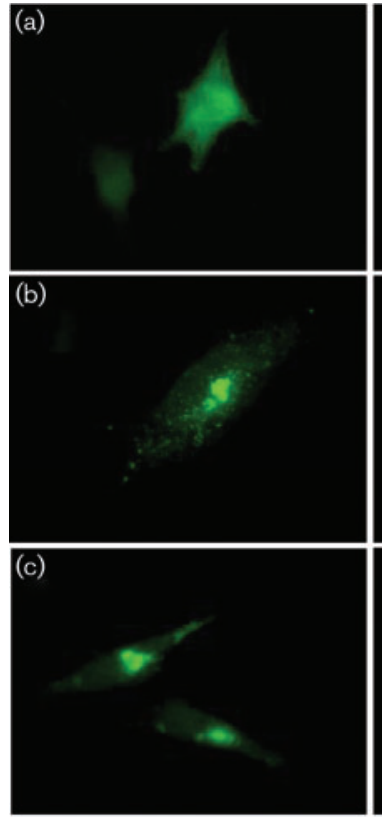

$7 \mathrm{C} 4 \mathrm{mAb}$
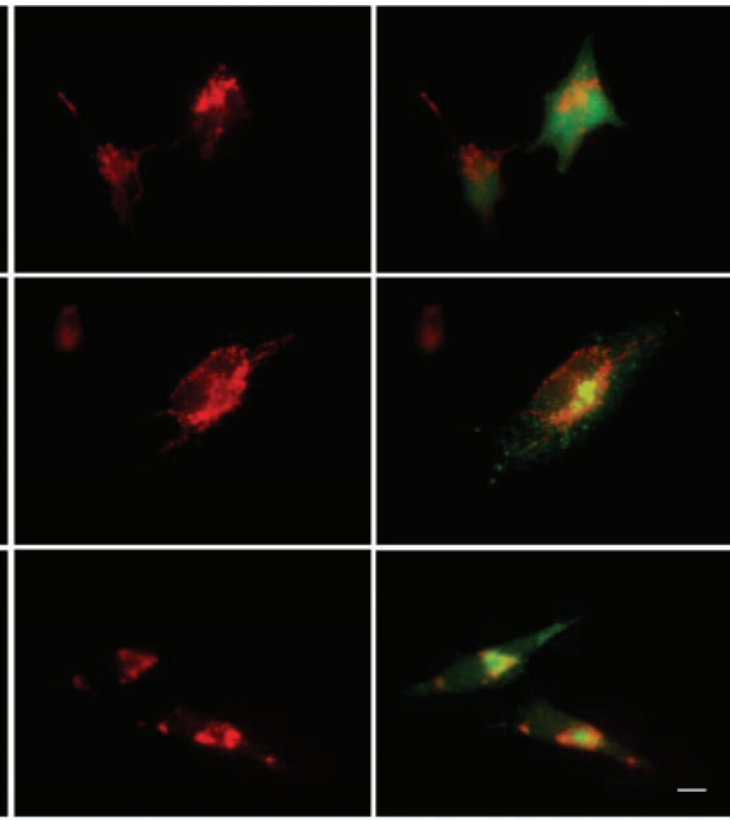

MitoTracker Red

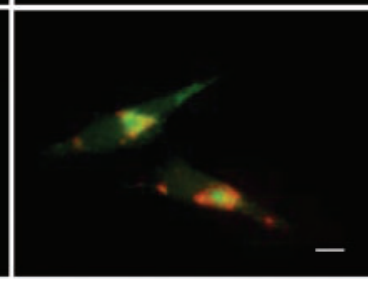

Merged
Fig. 6. Immunofluorescence showing the localization of additional PE_PGRS proteins. $\mathrm{RD}$ cells were transfected with PE_PGRS1 (a), PE_PGRS18 (b) and PE_PGRS24 (c). Cells stained with the PE_PGRS33-specific $\mathrm{mAb} 7 \mathrm{C} 4.1 \mathrm{~F} 7$ (left) and the mitochondriaspecific dye MitoTracker Red (centre) and the merged images (right) are shown. Magnification, $\times 1000$; bar, $10 \mu \mathrm{m}$. all constructs containing the PGRS domain of PE_PGRS1 had a diffuse pattern of fluorescence, whereas constructs containing the PGRS domain of PE_PGRS33 localized to the mitochondria of host cells irrespective of what PE domains or linker regions were present (Supplementary Fig. S3). Therefore, the specificity of $M$. tuberculosis PE_PGRS33 protein for the mitochondria of host cells is related to its PGRS domain.

\section{DISCUSSION}

The mechanisms of pathogenesis for M. tuberculosis are not well understood and the roles of individual proteins in the virulence of this pathogen have not been clearly defined. One molecule that has been implicated in M. tuberculosis pathogenesis is the PE_PGRS33 protein (Brennan et al., 2001; Balaji et al., 2007). In this study, we have investigated the localization of the M. tuberculosis PE_PGRS33 protein in transfected eukaryotic cells and have demonstrated that it co-localized to the mitochondria. Other bacterial proteins have also recently been found to associate with the mitochondria of host cells and in a few cases, a mitochondrial targeting sequence (MTS) with a putative cleavage signal is present at the $\mathrm{N}$-terminal end of these proteins (Kozjak-Pavlovic et al., 2008; Nougayrède \& Donnenberg, 2004; Papatheodorou et al., 2006). However, in other cases, bacterial proteins are targeted to the mitochondria without a discernible MTS, such as the Neisseria gonorrhoeae PorB protein which was shown to be imported into the host mitochondria by the same pathway used for the mitochondrial porin VDAC (Müller et al., 2002). Since no mitochondrial targeting sequences were identified in PE_PGRS33, several genetic constructs were prepared to investigate the roles of its different regions in this targeting phenomenon. Using these fusions, we showed that the PE domain of PE_PGRS33 is not required for co-localization to the mitochondria of host cells. Additionally, a construct where the PE domain was removed entirely (linker-PGRS) still localized to the mitochondria, although not as efficiently. In contrast, the 41 amino acid linker region situated between the PE and the PGRS domains of PE_PGRS33 was found to be essential to this phenomenon. Indeed, removing this linker abolished this localization, but adding it to MaPE-PGRS as well as replacing it with the linker from PE_PGRS1 allowed the resulting constructs to localize to the mitochondria of transfected host cells. Although these results show the importance of the linker region in this localization phenomenon, it does not answer the question of whether a specific localization signal is present in this segment or if the linker region is simply necessary for the proper translocation or folding of the PE_PGRS33 protein. Finally, although the PGRS domain by itself was not found to be sufficient for localization to the mitochondria, additional information on its importance was obtained by studying the chimeras where the different regions of PE_PGRS33 and PE_PGRS1 were systematically exchanged. Transfection of those chimeras into eukaryotic cells revealed that only the constructs with the PGRS domain of PE_PGRS33 localized to the mitochondria. These results indicate that the PGRS domain of PE_PGRS33 is able to target the mitochondria specifically whereas the PGRS domain of PE_PGRS1 is not, despite the fact that they are $55 \%$ identical. Protein sequence analysis using the SVMtm program revealed that the PGRS domain of PE_PGRS33 is more hydrophobic and has seven putative transmembrane segments not found in the PGRS domain 
(a)

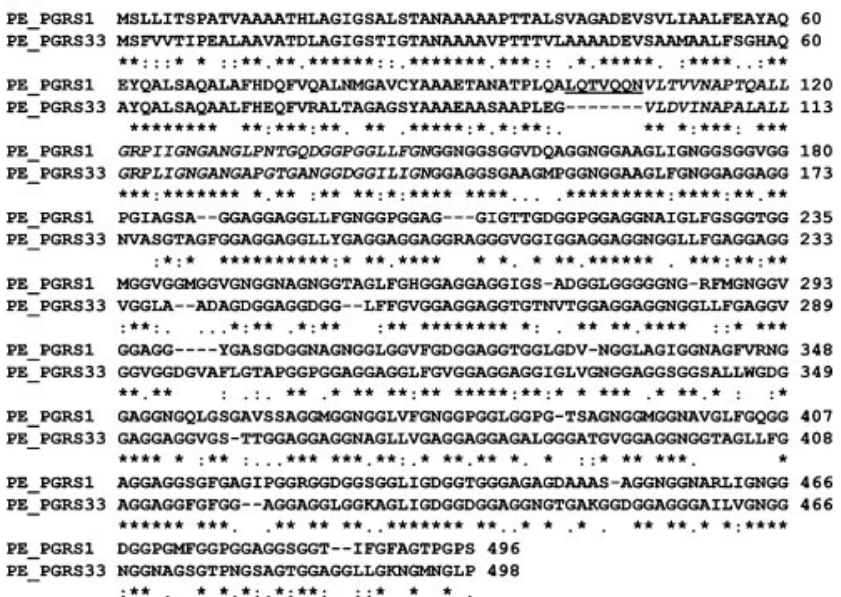

(b)

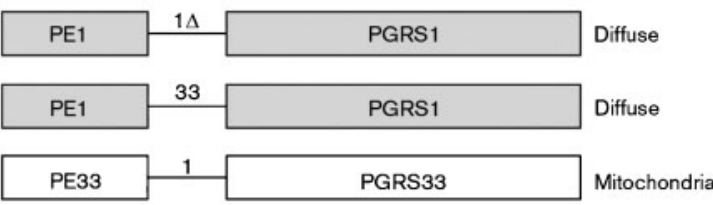

(c)

\begin{tabular}{|c|c|c|c|}
\hline РЕЗ3 & 33 & PGRS1 & Diffuse \\
\hline PЕ33 & 1 & PGRS1 & Diffuse \\
\hline PE1 & 3 & PGRS33 & Mitochondria \\
\hline PE1 & 1 & PGRS33 & Mitochondria \\
\hline
\end{tabular}

Fig. 7. PE_PGRS1 and PE_PGRS33 sequence comparison and constructed chimeras. (a) Protein sequence alignment of PE_PGRS1 and PE_PGRS33 showing the similarities between their PE domains (amino acids 1-100, $62 \%$ homology), their linker regions (italic type, $70 \%$ homology) and PGRS domains (remaining amino acids, $55 \%$ homology). The additional 7 amino acids found in PE_PGRS1 but not PE_PGRS33 are underlined. (b) Schematic of the constructs in which the extra 7 amino acids of PE_PGRS1 were removed and the linker regions of PE_PGRS1 and PE_PGRS33 were swapped, and their localization in transfected RD cells. (c) Schematic of the chimeras in which the PE domains, linker regions and PGRS domains of PE_PGRS1 and PE_PGRS33 were systematically swapped and their localization in transfected RD cells.

of PE_PGRS1 (Yuan et al., 2004). These sequence differences may explain how PE_PGRS33 is able to associate with the mitochondria of host cells. It is possible that the linker region of PE_PGRS33 is important for its proper translocation to the mitochondria, whereas the PGRS domain with its putative transmembrane segments actually binds to these organelles via membrane intercalating mechanisms. While the mechanisms associated with mitochondrial binding of PE_PGRS33 are being elicited, the reasons for the failure of PE_PGRS1 to interact with mitochondria remain uncertain. It is possible that sequences within PE_PGRS1 could inhibit mitochondrial interactions. Studies using a PE_PGRS33-PGRS1 construct should address whether the presence of PGRS1 sequences prohibits mitochondrial binding.

Another important aspect of this study was our investigation of a possible correlation between localization to the mitochondria and the induction of cell death. An earlier study showed that a recombinant $M$. smegmatis strain expressing PE_PGRS33 induced necrosis in BMMO, whereas others have more recently reported that PE_PGRS33 induced apoptosis of eukaryotic cells (Balaji et al., 2007; Basu et al., 2007; Dheenadhayalan et al., 2006a). To measure and characterize the cell death induced by the different constructs prepared in this study, stable inducible cell lines were created using the T-REx inducible mammalian expression system. Using these cell lines, a direct correlation was observed between localization to the host cell mitochondria and the induction of cell death using a combination of Hoechst 33342/YO-PRO-1/PI staining and flow cytometry.

Importantly, these flow cytometry studies have also identified the mechanisms of cell death that may be associated with the localization of the PE_PGRS33 protein to the mitochrondria. Historically, three types of cell death - apoptosis, autophagy, and necrosis - have been distinguished in mammalian cells using morphological criteria (Golstein \& Kroemer, 2007). Our flow cytometry data indicate that apoptosis is a primary mechanism of cell death after PE_PGRS33-mitochrondrial interactions. Moreover, preliminary RT-PCR results have shown that the expression of FADD (Fas-associated death-receptor domain) and caspase 8 is upregulated after the induction of PE_PGRS33 synthesis (N. Cadieux, unpublished results) and, therefore, suggest that apoptosis probably occurs via the extrinsic or death-receptor pathway. Further studies will focus more precisely on defining the relevant apoptotic pathway using RT-PCR and caspase inhibitor assays. Interestingly, although several of these constructs induced apoptosis, only the wild-type PE_PGRS33 protein also induced a significant amount of primary necrosis $(24.3 \%$ at $72 \mathrm{~h}$ compared with 4.5 and $5.3 \%$ for the other two constructs). Since the only difference between PE_PGRS33 and MaPE(linker)PGRS is the PE domain being replaced by MaPE, these results suggest a very important role for the PE domain of PE_PGRS33. Indeed, while it had minimal impact on mitochrondrial localization, the PE domain seems to be important for the induction of primary necrosis. Surprisingly, other investigators have not detected the induction of necrosis following exposure of cells to PE_PGRS33. For example, Balaji et al. (2007) found no necrosis in Jurkat cells transfected with PE_PGRS33 fused to EGFP. In a related study, when recombinant PE_PGRS33 protein was delivered extracellularly to RAW264.7 cells, the induction of necrosis by PE_PGRS33 also was not observed (Basu et al., 2007). It is unclear why other investigators have not detected PE_PGRS33mediated necrosis, but different levels of expression, varying detection methods and the use of different cell 
lines with distinct sensitivities to necrosis may explain the disparate results.

Assuming the PE_PGRS33 protein does induce cell death during natural infection, it is presently unclear how this protein can translocate from the bacterium, which is thought to be sequestered within phagosomes, to the mitochondria of infected cells. However, advances in the field of mycobacteriology are providing clues to help answer this question. Recent studies have demonstrated that pathogenic species of mycobacteria such as $M$. tuberculosis, M. leprae and M. marinum are capable of phagosomal escape and translocation into the cytosol of infected antigen-presenting cells (McDonough et al., 1993; Stamm et al., 2003; van der Wel et al., 2007). Furthermore, a new type of secretion system, the type VII secretion system, has recently been described in pathogenic mycobacteria, which allows them to secrete different potential virulence factors that do not have a typical signal sequence, including proteins from the PE and PPE families (Abdallah et al., 2006, 2007, 2009). It is therefore conceivable that the PE_PGRS33 protein can interact with the mitochondria of host cells during natural infection after phagosomal escape or via a novel secretion system. Clearly, future experiments will be needed to ascertain whether PE_PGRS33 is secreted and to confirm the potential role of PE_PGRS33 secretion in the induction of cell death. Considering the importance of primary necrosis of infected host cells in the dissemination of $M$. tuberculosis during natural infection, the results of these future studies could further implicate PE_PGRS33 in the pathogenesis of tuberculosis.

\section{ACKNOWLEDGEMENTS}

We would like to thank Veerabadran Dheenadhayalan, Karen Meysick and Jackie Muller for invaluable discussions during this study. This work was supported in part by a grant from the National Vaccine Program Office of the Department of Health and Human Services to M. J. B.

\section{REFERENCES}

Abarca-Rojano, E., Rosas-Medina, P., Zamudio-Cortéz, P., Mondragón-Flores, R. \& Sánchez-Garcia, F. J. (2003). Mycobacterium tuberculosis virulence correlates with mitochondrial cytochrome $c$ release in infected macrophages. Scand J Immunol 58, 419-427.

Abdallah, A. M., Verboom, T., Hannes, F., Safi, M., Strong, M., Eisenberg, D., Musters, R. J., Vandenbroucke-Grauls, C. M., Applemelk, B. J. \& other authors (2006). A specific secretion system mediates PPE41 transport in pathogenic mycobacteria. Mol Microbiol 62, 667-679.

Abdallah, A. M., Gey van Pittius, N. C., Champion, P. A. D., Cox, J., Luirink, J., Vandenbroucke-Grauls, C. M., Applemelk, B. J. \& Bitter, W. (2007). Type VII secretion - mycobacteria show the way. Nat Rev Microbiol 5, 883-891.

Abdallah, A. M., Verboom, T., Weerdenburg, E. M., Gey van Pittius, N. C., Mahasha, P. W., Jiménez, C., Parra, M., Cadieux, N., Brennan, M. J. \& other authors (2009). PPE and PE_PGRS proteins of
Mycobacterium marinum are transported via the type VII secretion system ESX-5. Mol Microbiol 73, 329-340.

Balaji, K. N., Goyal, G., Narayana, Y., Srinivas, M., Chaturvedi, R. \& Mohammad, S. (2007). Apoptosis triggered by Rv1818c, a PE family gene from Mycobacterium tuberculosis is regulated by mitochondrial intermediates in T cells. Microbes Infect 9, 271-281.

Basu, S., Pathak, S. K., Banerjee, A., Pathak, S., Bhattacharyya, A., Yang, Z., Talarico, S., Kundu, M. \& Basu, J. (2007). Execution of macrophage apoptosis by PE_PGRS33 of Mycobacterium tuberculosis is mediated by Toll-like receptor 2-dependent release of tumor necrosis factor- $\alpha$. J Biol Chem 282, 1039-1050.

Brennan, M. J. \& Delogu, G. (2002). The PE multigene family: a 'molecular mantra' for mycobacteria. Trends Microbiol 10, 246-249.

Brennan, M. J., Delogu, G., Chen, Y., Bardarov, S., Kriakov, J., Alavi, M. \& Jacobs, W. R. (2001). Evidence that mycobacterial PE_PGRS proteins are cell surface constituents that influence interactions with other cells. Infect Immun 69, 7326-7333.

Brennan, M. J., Gey van Pittius, N. C. \& Espitia, C. (2005). The PE and PPE multigene families of Mycobacteria. In Tuberculosis and the Tubercle Bacillus, pp. 513-525. Edited by S. T. Cole, K. D. Eisenach, D. N. McMurray \& W. R. Jacobs. Washington, DC: American Society for Microbiology.

Cadieux, N. \& Kadner, R. J. (1999). Site-directed disulfide bonding reveals an interaction site between energy-coupling protein TonB and BtuB, the outer membrane cobalamin transporter. Proc Natl Acad Sci U S A 96, 10673-10678.

Camus, J. C., Pryor, M., Médigue, C. \& Cole, S. T. (2002). Reannotation of the genome sequence of Mycobacterium tuberculosis H37Rv. Microbiology 148, 2967-2973.

Chen, M., Gan, H. \& Remold, H. G. (2006). A mechanism of virulence: virulent Mycobacterium tuberculosis strain H37Rv, but not attenuated H37Ra, causes significant mitochondrial inner membrane disruption in macrophages leading to necrosis. J Immunol 176, 3707-3716.

Cole, S. T., Brosch, R., Parkhill, J., Garnier, T., Churcher, C., Harris, D., Gordon, S. V., Eiglmeier, K., Gas, S. \& other authors (1998). Deciphering the biology of Mycobacterium tuberculosis from the complete genome sequence. Nature 393, 537-544.

Delogu, G., Pusceddu, C., Bua, A., Fadda, G., Brennan, M. J. \& Zanetti, S. (2004). Rv1818c-encoded PE_PGRS protein of Mycobacterium tuberculosis is surface exposed and influences bacterial cell structure. Mol Microbiol 52, 725-733.

Delogu, G., Sanguinetti, M., Pusceddu, C., Bua, A., Brennan, M. J., Zanetti, S. \& Fadda, G. (2006). PE_PGRS proteins are differentially expressed by Mycobacterium tuberculosis in host tissues. Microbes Infect 8, 2061-2067.

Dheenadhayalan, V., Delogu, G. \& Brennan, M. J. (2006a). Expression of the PE_PGRS33 protein in Mycobacterium smegmatis triggers necrosis in macrophages and enhanced mycobacterial survival. Microbes Infect 8, 262-272.

Dheenadhayalan, V., Delogu, G., Sanguinetti, M., Fadda, G. \& Brennan, M. J. (2006b). Variable expression patterns of Mycobacterium tuberculosis PE_PGRS genes: evidence that PE_PGRS16 and PE_PGRS26 are inversely regulated in vivo. J Bacteriol 188, 3721-3725.

Duan, L., Gan, H., Golan, D. E. \& Remold, H. G. (2002). Critical role of mitochondrial damage in determining outcome of macrophage infection with Mycobacterium tuberculosis. J Immunol 169, 5181-5187.

Espitia, C., Laclette, J. P., Mondragón-Palomino, M., Amador, A., Campuzano, J., Martens, A., Singh, M., Cicero, R., Zhang, Y. \& Moreno, C. (1999). The PE-PGRS glycine-rich proteins of Mycobacterium tuberculosis: a new family of fibronectin-binding proteins? Microbiology 145, 3487-3495. 
Flores, J. \& Espitia, C. (2003). Differential expression of PE and PE_PGRS genes in Mycobacterium tuberculosis strains. Gene 318, 75-81.

Fratazzi, C., Arbeit, R. D., Carini, C. \& Remold, H. G. (1997). Programmed cell death of Mycobacterium avium serovar 4-infected human macrophages prevents the Mycobacteria from spreading and induces mycobacterial growth inhibition by freshly added, uninfected macrophages. J Immunol 158, 4320-4327.

Gan, H., He, X., Duan, L., Mirabile-Levens, E., Kornfeld, H. \& Remold, H. G. (2005). Enhancement of antimicrobial activity of macrophages by stabilization of inner mitochondrial membrane potential. $J$ Infect Dis 191, 1292-1300.

Gey van Pittius, N. C., Sampson, S. L., Lee, H., Kim, Y., van Helden, P. D. \& Warren, R. M. (2006). Evolution and expansion of the Mycobacterium tuberculosis PE and PPE multigene families and their association with the duplication of the ESAT- 6 (esx) gene cluster regions. BMC Evol Biol 6, 95.

Golstein, P. \& Kroemer, G. (2007). Cell death by necrosis: toward a molecular definition. Trends Biochem Sci 32, 37-43.

Gordon, S. V., Eiglmeier, K., Brosch, R., Garnier, T., Honoré, N., Barrell, B. \& Cole, S. T. (1999). Genomics of Mycobacterium tuberculosis and Mycobacterium leprae. In Mycobacteria: molecular biology and virulence, pp. 93-109. Edited by C. Ratledge \& J. Dale. Oxford: Blackwell Science Ltd.

Hillion, J. A., Takahashi, K., Maric, D., Ruetzler, C., Barker, L. J. \& Hallenbeck, M. (2005). Development of an ischemic tolerance model in a PC12 cell line. J Cereb Blood Flow Metab 25, 154-162.

Karboul, A., Mazza, A., Gey van Pittius, N. C., Ho, J. L., Brousseau, R. \& Mardassi, H. (2008). Frequent homologous recombination events in Mycobacterium tuberculosis PE/PPE multigene families: potential role in antigenic variability. $J$ Bacteriol 190, 7838-7846.

Kozjak-Pavlovic, V., Ross, K. \& Rudel, T. (2008). Import of bacterial pathogenicity factors into mitochondria. Curr Opin Microbiol 11, 9-14.

McDonough, K. A., Kress, Y. \& Bloom, B. R. (1993). Pathogenesis of tuberculosis: interactions of Mycobacterium tuberculosis with macrophages. Infect Immun 61, 2763-2773.

Müller, A., Rassow, J., Grimm, J., Machuy, N., Meyer, T. F. \& Rudel, T. (2002). VDAC and the bacterial porin PorB of Neisseria gonorrhoeae share mitochondrial import pathways. EMBO J 21, 1916-1929.

Nougayrède, J.-P. \& Donnenberg, M. S. (2004). Enteropathogenic Escherichia coli EspF is targeted to mitochondria and is required to initiate the mitochondrial death pathway. Cell Microbiol 6, 10971111.

Papatheodorou, P., Domańska, G., Öxle, M., Mathieu, J., Selchow, O., Kenny, B. \& Rassow, J. (2006). The enteropathogenic Escherichia coli (EPEC) Map effector is imported into the mitochondrial matrix by the TOM/Hsp70 system and alters organelle morphology. Cell Microbiol 8, 677-689.

Park, J. S., Tamayo, M. H., Gonzalez-Juarrero, M., Orme, I. M. \& Ordway, D. J. (2006). Virulent clinical isolates of Mycobacterium tuberculosis grow rapidly and induce cellular necrosis but minimal apoptosis in murine macrophages. J Leukoc Biol 79, 80-86.
Parra, M., Cadieux, N., Pickett, T., Dheenadhayalan, V. \& Brennan, M. J. (2006). A PE protein expressed by Mycobacterium avium is an effective T-cell immunogen. Infect Immun 74, 786-789.

Porcelli, S. A. \& Jacobs, W. R., Jr (2008). Tuberculosis: unsealing the apoptotic envelope. Nat Immunol 9, 1101-1102.

Poulet, S. \& Cole, S. T. (1995). Characterization of the highly abundant polymorphic GC-rich-repetitive sequence (PGRS) present in Mycobacterium tuberculosis. Arch Microbiol 163, 87-95.

Ramakrishnan, L., Federspiel, N. A. \& Falkow, S. (2000). Granulomaspecific expression of Mycobacterium virulence proteins from the glycine-rich PE-PGRS family. Science 288, 1436-1439.

Schaible, U. E., Winau, F., Sieling, P. A., Fischer, K., Collins, H. L., Hagens, K., Modlin, R. L., Brinkmann, V. \& Kaufmann, S. H. (2003). Apoptosis facilitates antigen presentation to $\mathrm{T}$ lymphocytes through MCH-I and CD1 in tuberculosis. Nat Med 9, 1039-1046.

Singh, P. P., Parra, M., Cadieux, N. \& Brennan, M. J. (2008). A comparative study of host response to three Mycobacterium tuberculosis PE_PGRS proteins. Microbiology 154, 3469-3479.

Stamm, L. M., Morisaki, J. H., Gao, L. Y., Jeng, R. L., McDonald, K. L., Roth, R., Takeshita, S., Heuser, J., Welch, M. D. \& Brown, E. J. (2003). Mycobacterium marinum escapes from phagosomes and is propelled by actin-based motility. J Exp Med 198, 1361-1368.

Talaat, A. M., Lyons, M. R., Howard, S. T. \& Johnston, S. A. (2004). The temporal expression profile of Mycobacterium tuberculosis infection in mice. Proc Natl Acad Sci U S A 101, 4602-4607.

Talarico, S., Cave, M. D., Marrs, C. F., Foxman, B., Zhang, L. \& Yang, Z. (2005). Variation of the Mycobacterium tuberculosis PE_PGRS33 gene among clinical isolates. J Clin Microbiol 43, 4954-4960.

Talarico, S., Cave, M. D., Foxman, B., Marrs, C. F., Zhang, L., Bates, J. H. \& Yang, Z. (2007). Association of Mycobacterium tuberculosis PE_PGRS33 polymorphism with clinical and epidemiological characteristics. Tuberculosis (Edinb) 87, 338-346.

Talarico, S., Zhang, L., Marrs, C. F., Foxman, B., Cave, M. D., Brennan, M. J. \& Yang, Z. (2008). Mycobacterium tuberculosis PE_PGRS16 and PE_PGRS26 genetic polymorphism among clinical isolates. Tuberculosis (Edinb) 88, 283-294.

Towbin, H., Staehlin, T. \& Gordon, J. (1979). Electrophoretic transfer of proteins from polyacrylamide gels to nitrocellulose sheets: procedure and some applications. Proc Natl Acad Sci U S A 76, 4350-4354.

van der Wel, N., Hava, D., Houben, D., Fluitsma, D., van Zon, M., Pierson, J., Brenner, M. P. \& Peters, J. (2007). M. tuberculosis and M. leprae translocate from the phagolysosome to the cytosol in myeloid cell. Cell 129, 1287-1298.

WHO (2008). Global tuberculosis control - surveillance, planning, financing. In World Health Organization report. Geneva: World Health Organization. http://www.who.int/tb/publications/global_ report/2008/en/index.html

Yuan, Z., Mattick, J. S. \& Teasdale, R. D. (2004). SVMtm: support vector machines to predict transmembrane segments. J Comput Chem 25, 632-636.

Edited by: G. R. Stewart 